\title{
Local Current-activated Growth of Nanometric Nickel Pillars During In situ STM-TEM Experiments
}

\author{
Klaus van Benthem, Jorgen F. Rufner, Cecile S. Bonifacio, Troy B. Holland, Ricardo H.R. \\ Castro
}

Department of Chemical Engineering and Materials Science, One Shields Avenue University of California, Davis CA 95616

Local directional control for the growth of nanomaterials remains a great challenge faced by the electronic community to develop novel three-dimensional devices with enhanced performance. The architecture of vertical electronics cannot be obtained with many current setups for manufacturing and therefore requires the development of new strategies for synthesis. One alternative may be the local consolidation, i.e. sintering, of nanoparticles into specific geometrical shapes by application of electrical fields or currents.

Sintering of nanometric powder particles can be considered as competition of densification and crystal growth. We have used in situ transmission electron microscopy (TEM) to study the sintering behavior of nanometric nickel particles through current-assisted densification. Small agglomerates of Ni powder particles suspended on holey amorphous carbon films were directly contacted with a scanning tunneling microscopy (STM) tip and exposed to constant bias stress (see Figure 1). In situ TEM experiments were carried out with a Nanofactory double-tilt STMTEM sample holder inserted into a Jeol JEM 2100F/Cs scanning transmission electron microscope operated at $200 \mathrm{keV}$.

Morphological changes of the particles were video taped through in situ STEM imaging using bright field field and high angle annular dark field detectors. The resulting current across the particle agglomerate was recorded as a function of time during image acquisition. After an initial incubation time due to electric field-induced dielectric breakdown of surface oxide layers, we observed the onsets of densification of the particle agglomerate on the carbon support film while particles that were in contact with the STM tip and extended into the vacuum coalesced and exhibited grain growth. As a consequence, a nickel nanorod started growing between the STM tip and the particle agglomerate (see Figure 2). During the observed Czochralski-type growth, planar defects were introduced into the growing nanostructure in the direction perpendicular to the growth direction. The growth defects are correlated with abrupt changes in resulting currents, i.e. specific Ohmic resistances. Preliminary characterizations of the growth conditions suggest that grain growth of nickel is kinetically favored over densification of the particle agglomerate. Initial systematic experimental studies furthermore indicate that growth of the nanostructure is likely facilitated by electromigration of nickel. More detailed analyses of the growth mechanisms are currently underway and will be discussed during the presentation.

[1] Morsi, K., et al., Scripta Materialia, 2009. 60(9): p. 745-748

[2] German, R.M., Sintering Theory and Practice 1996, New York: John Wiley \& Sons, Inc.

[3] Bonifacio, C.S., et al., Applied Physics Letters, 2012. 101: p. 093107 
[4] The authors acknowledge funding from a University of California Laboratory Fee Grant (\#12-LR-238313), the US Department of Defense, Army Research Office (grant \#W911nf12-1-0491-0), and the Office of Naval Research (grant \#N00014-10-1-0632).

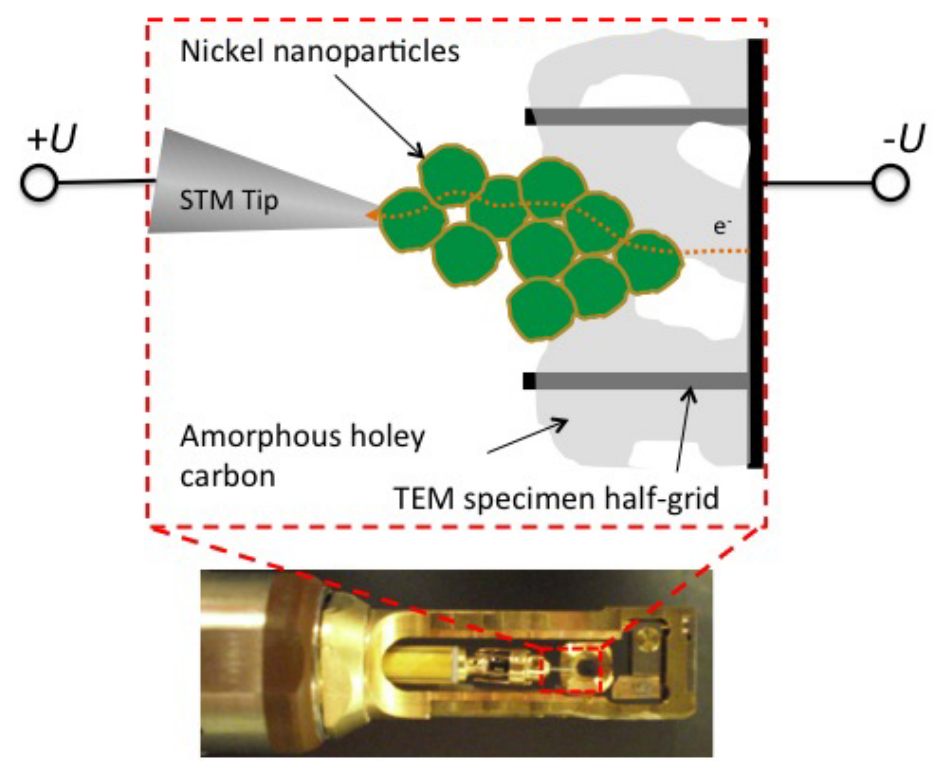

Figure 1: Sketch of the experimental geometry. Ni nanoparticles with an average diameter of 20 $\mathrm{nm}$ were drop-casted on amorphous holey carbon films placed on TEM half-grids. An STM tip mounted on a Nanofactory sample holder was brought in contact with particle agglomerates for electrical contacting.
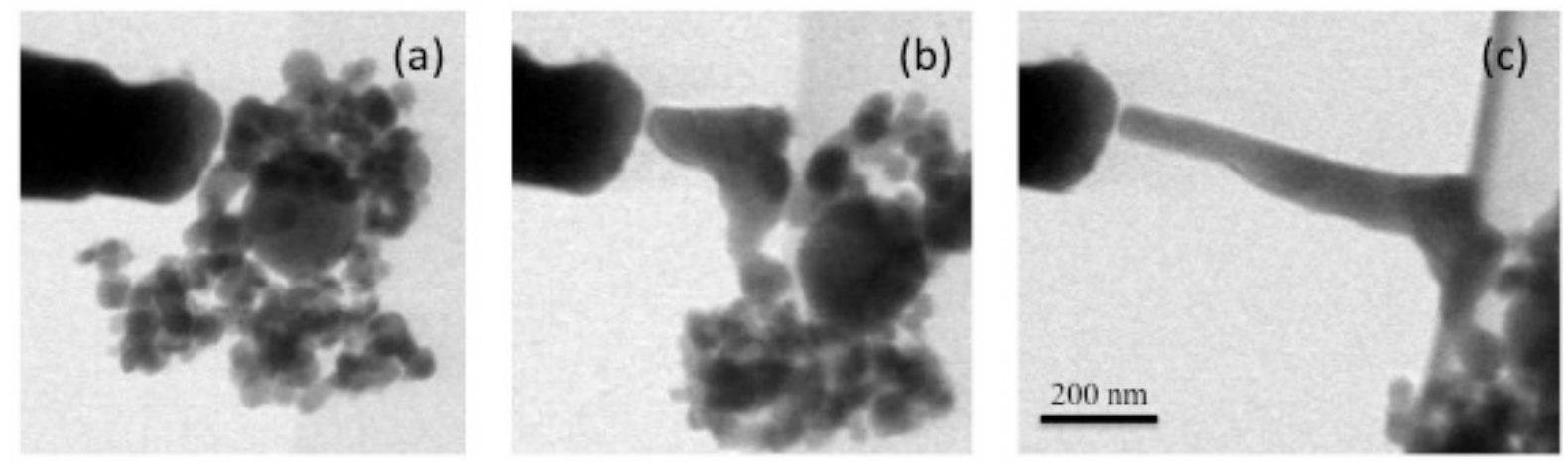

Figure 2: A positive electrical bias applied to the STM tip enabled consolidation of Ni nanoparticles that results in directional growth of a nanopillar. 